Kölbchen $a$ wird auf circa $1 / 4$ mit Benzin gefüllt und in warmes Wasser gestellt. Dadurch destillirt das Benzin nach $b$ über. Stellt man dann $a$ in kaltes Wasser, so steigt das Benzin nach $a$ zurück und nimmt das lösliche Fett mit.

Nach drei- bis viermaligem Destilliren ist die Substanz fettfrei, die Röhre wird bei $110^{\circ}$ getrocknet und gewogen. Die ganze Untersuchung mit vier Wägungen ist in $3 / 4$ Stunden gut beendet.

\title{
Ueber die quantitative Bestimmung des Nicotins im Tabak.
}

Von Dr. J. Skal weit in Hannover.

In Folge einer grösseren Arbeit, welche ich in Gemeinschaft mit Herrn E. Wenderoth, dem Erfinder eines Apparats zur Entfernung des Nicotins aus schlechtfermentirten Tabaken, unternommen, hatte ich in den letzten Jahren sehr häufig Gelegenheit, Nicotinbestimmungen auszuführen und ausführen zu lassen. Die AnzahI dieser Analysen beläuft sich auf mehrere hunderte und die Erfahrungen, welche dabei gesammelt wurden, sind so vielseitig, dass es angebracht erscheint, schon vor Erscheinen der oben erwähnten grösseren Arbeit in den "Annalen", an dieser Stelle über die angewandten analytischen Methoden und deren Brauchbarkeit kurz zu referiren.

Der erste, welcher das Nicotin quantitativ in Tabak zu bestimmen unternahm, war Th. Schloesing im Jahre 1846. ${ }^{1}$

Seine Methode (pag. $238 \mathrm{ff}$.) besteht darin, das Nicotin mittelst Ammoniak frei zu machen und in Aether aufzunehmen, den Aether durch Erhitzen von Ammoniak zu befreien und den Rückstand mit Normal-Schwefelsäure zu titriren.

$\mathrm{Zu}$ diesem Zweck werden $10 \mathrm{~g}$. des fern gepulverten Tabaks lufttrocken in eine $20 \mathrm{~mm}$. weite Röhre derart eingetragen, dass nach jedem eingetragenen Löffelchen Tabak ein Tropfen Ammoniakflüssigkeit eingegossen und diese Röhre einerseits mit einem Liebig'schen Kühler und andererseits mit einem seitlich tubulirten Kölbchen in Verbindung gebracht wird. Das letztere Kölbchen

1) Ann. d. Chim. et Phys. 1847. Tom. 19. S. 230. 
von einem Inhalt von ca. $150-200$ C.C. verbindet man nach der Beschickung mit ca. 30 C.C. Aether an seiner Halsöffnung mit der anderen (höher liegenden) Seite des Liebig'schen Kühlers und erwärmt. Der Aether steigt so in den Kühler, condensirt sich daselbst, tröpfelt auf den mit Ammoniak befeuchteten Tabak, fliesst davon mit Nicotin und Ammoniak beschwert wieder ab in das Kölbchen und macht denselben Weg von neuem, indem er stets neue Mengen Nicotin in das Kölbchen nimmt, bis die gesammte Menge des Nicotins sich als ätherische Lösung mit etwas Ammoniak im Kölbchen befindet. In 2-4 Stunden soll die Auslaugung des Tabaks beendigt sein. Man überzeugt sich davon durch Probenahme einiger Tropfen der ablaufenden Aetherlösung auf einem Uhrglas und Verdunsten derselben in mässiger Wärme. Verbreitet sich hierbei nicht der geringste Geruch nach Nicotin, so ist die Auslaugung beendet.

Man entfernt nun die mit Tabak gefüllte Röhre und bringt statt derselben eine Vorlage an den Kühler. Wird jetzt weiter erhitzt, so gehen zunächst stark ammoniakalische Aetherd̈ämpfe über - allein bald ist der übergehende Aetber vollkommen neutral und es befindet sich im Rückstand nur noch Nicotin und kein Ammoniak. Ist dieser Punkt erreicht, giesst man in eine Porzellanschale $a b$, wäscht mit Aether aus, lässt an freier Luft verdunsten und titrirt mit Lackmuspapierstreifen als Indicator.

Die Schloesing'sche Methode wird auch beute noch in den neuesten Lehrbüchern und Handbüchern als die beste empfohlen, und wenn auch andere Vorschläge in der Literatur sich vorfinden, so schien es doch zunächst nothwendig, diese Methode anzuwenden und zugleich anf ihre Brauchbarkeit zu prüfen.

Es ist mir nach längerer Erfahrung nicht möglich, in das allgemeine Lob über die Brauchbarkeit derselben einzustimmen. Zunächst ist es eine sehr schwierige Aufgabe, in einer harzigen schmierigen Masse, wie man sie als Tabaksauszug nach dem Verdunsten des Aethers erhält, den Sättigungspnnkt mit Lackmuspapier auch nur einigermaassen zuverlässig zu bestimmen - dann aber ist auch der Punkt, wann die Auslaugung vollständig erfolgt ist, nie genau zu treffen. Selbst nach 3-4tägigem Ausziehen, (täglich 12 Stunden lang) wurden am 4. resp. 5. Tage noch deutlich gefärbte Lösungen erhalten, namentlich wenn nach dem Stehen in der Nacht einige Tropfen Ammoniak zu dem Tabak hinzugefügt waren, ehe der Apparat wieder in Betrieb gesetzt wurde. 
Der erste Uebelstand hat mich bewogen, die Schloesing'sche Methode zu modificiren und stets die gewonnenen Auszüge nicht direct zu titriren, sondern mit Kalilauge zu destilliren. Man hat hierbei jedoch wohl zu bedenken, dass bei Anwendung wässerigen Ammoniaks zur Befeuchtung des 'Tabaks stets Ammoniaksalze aus dem Tabak in Lösung gehen und dass diese einen verhängnissvollen Einfluss auf die Genauigkeit der Analyse ausüben müssen, selbst wenn sie nur in geringer Menge vorhanden sein sollten. Das Aequivalent des Nicotins ist nämlich etwa $10 \mathrm{mal}$ so hoch als das des Ammoniaks und jedes Zehntel-Procent von letzterem erhöht das Resultat um ebensoviel ganze Procente an Nicotin. Um seiner Bestimmung daher einigermaassen sicher zu sein, ist es stets erforderlich, das Destillat nach der Bestimmung einzudampfen, mit $90 \%$ tigem Alkohol auszuziehen und den Rückstand nach dem Lösen in Wasser auf Schwefelsäure resp. Ammoniumsulfat zu untersuchen.

Eine solche Prüfung ist jedoch umständlich und macht es wünschenswerth, statt des wässerigen Ammoniaks eine andere Base zur Tabakszersetzung einzuführen, wodnrch die Isolirung von Ammonsalzen weniger wahrscheinlich wird.

Es wurde daher mit Kalilauge in wässeriger und in alkoholischer Lösung operirt und sind dabei zwar viel weniger ammoniakhaltige, allein nie ganz ammoniakfreie Destillate erzielt, so dass es auch hier stets erforderlich wurde, das Destillat einzudampfen und in schwefelsaures Ammoniak und Nicotinsulfat zu zerlegen.

Die besten Resultate wurden erhalten, wenn der Tabak sogleich nach dem Trocknen noch warm mit einer concentrirten alkoholischen Kalilauge angefeuchtet und sofort mit Aether übergossen wurde. Es waren dann nur so geringe Spuren von Ammoniak im Destillat nachzuweisen, dass dieselben ohne Bedenken vernachlässigt werden konnten.

Statt des Ausziehens mit Aether in einer Schloesing'schen Röhre und doppelt tubulirtem Kolben, statt dessen ich mit bestem Erfolg ein gewöhnliches Fractionirkölbchen anwandte, habe ich auch die Fettextractionsapparate nach Soxhlet und nach Tollens erprobt, kann aber nicht sagen, dass sie vor der einfachen Schloesing'schen Anordnung irgend welchen Vorzug verdienen. Ebensowenig ist der Schiel'sche Apparat, aus zwei Flaschen bestehend, die abwechselnd in laues und kaltes Wasser gestellt werden, als handlich und zeitersparend zu empfehlen. 
J. Skalweit, Ueber die quantitative Bestimmung des Nicotins in Tabak. 39

Hat man einmal das directe Titriren aufgegeben und sich dazu entschlossen, die erhaltenen Extracte stets zu destilliren, so tritt sehr bald die Frage an den Analytiker, in welcher Weise dies am geeignetsten auszuführen ist.

Die Gefahr der Zersetzung des Nicotins durch Kalilauge in höherer Temperatur ist zweifellos vorhanden und mag daraus die in verschiedenen Laboratorien eingeführte und auch von mir versuchte Methode der Destillation mit Wasserdampf im Wasserdampfotrom sich hergeleitet haben.

Man unterhält zu diesem Zweck einen grossen Kolben mit destillirtem Wasser in heftigem Kochen und leitet die Dämpfe auf den Boden eines kleineren Kolbens, in welchem sich das alkalische Nicotinextract befindet. Die aus dem letzteren sich dabei entwickelnden Dämpfe werden in einen Kühler geleitet und aufgefangen. Nimmt die Menge des Wassers im kleinen Kolben zu sehr zu, so erwärmt man denselben mittelst einer kleinen Flamme und sotat die Destillation so Iange fort, als noch alkalische Reaction auftritt.

Ich will hier Jedermann ausdrïcklich vor der Anwendung dieser Mlethode warnen. Sie lässt stets im Stich und ist ganz unzuverlässig. Auch ist sie wegen der grossen Menge Destillat, die bei ihrer Anwendung erhalten wird, höchst unpractisch, da dieser Umstand nicht das scharfe Umschlagen der Lackmusreaction zu erkennen gestattet. Auch ist die Gefahr der Zersetzung von Nicotin durch Kalilauge viel geringer, als durch gespannte Wasserdämpfe. ${ }^{1}$ Man hat nur dafür Sorge zu tragen, dass die Concentration der Kalilauge nicht zu weit getrieben wird und die Temperatur nicht zu hoch steigt.

Jedenfalls erhält man gut übereinstimmende Zahlen, so wie man sich gewöhnt hat, mit gleichen Quantitäten Kalilauge von demselben specifischen Gewicht zu arbeiten und niemals die colossalen Abweichungen wie bei der Wasserdampfdestillation.

Ueberhaupt scheinen mir die Hauptfehlerquellen nicht auf dem Theil der Nicotinbestimmung zu liegen, welcher sich mit der Destillation des fertigen Extracts befasst. Denn ich habe wiederholt

1) Ich werde an einer anderen Stelle zeigen, dass Nicotin durch gespannte Wasserdämpfe von ca. 2 Atmosphären schon bei $110^{\circ} \mathrm{C}$. zersetzt wird in Körper, von denen einer Ammoniak, ein zweiter Kohlensäure ist. 
bei Analysen von fertigen Tabakslaugen, in denen die Bestimmung des Ammoniaks gleichzeitig mit der des Nicotins vorgenommen werden musste, und wo eine besondere Auslaugung und Präparation nicht erforderlich war, gefunden, dass die erhaltenen Zahlen fast immer von Anfang an gut stimmten und bei vergleichenden Analysen kaum $1 / 10 \%$ grosse Abweichungen ergaben.

Bedeutend grössere Abweichungen erhält man jedoch, wenn erst ein Auszug aus dem Tabak gemacht werden muss und nach einer der in den Lehrbüchern beschriebenen allgemein üblichen Methode verfahren wird. Diese Abweichungen sind nur dadurch zu erklären, dass die Behandlung mit Aether nicht lange genug erfolgt ist und kalter Aether nur äusserst langsam anf Tabak wirkt.

Ein ungleich besseres Mittel ist 98\% tiger Aethyl-Alkohol; derselbe löst in kurzer Zeit die Nicotinsulfate, namentlich in der Wärme, und kann ich allen Analytikern, denen es auf genaue Resultate ankommt, denselben zur Nicotinanalyse sehr empfehlen. Meine Methode ist nunmehr kurz folgende:

Der zu untersuchende Tabak wird bei $50^{\circ} \mathrm{C}$. auf einem regulirten Luftbad getrocknet, fein gepulvert, gesiebt und der Rückstand nach abermaliger Pulverung und Siebung zum Ganzen gemischt, so dass eine gleichmässige lufttrockene Durchschnittsprobe nach der Beendigung der Operation als feines Pulver vorliegt. Dasselbe wird in eine luftdicht geschlossene Flasche gebracht, in einer kleinen Probe der Feuchtigkeitsgehalt bei $100^{\circ}$ ermittelt und dieser auf der Flasche notirt. Von der so vorgerichteten Substanz wiegt man $20,25 \mathrm{~g}$. ab, befeuchtet mit $10 \mathrm{C}$. C. Normal-Schwefelsäure (1 C.C. $=0,049 \mathrm{~g}$. $\mathrm{SO}^{4} \mathrm{H}^{2}$ ) spült dieselben mit $200 \mathrm{C.C}$. $98 \%$ tigem Alkohol in einen Kolben und kocht 2 Stunden am Rückflusskühler, lässt erkalten, giesst in einen 250 C.C. fassenden Messkolben, spült mit absolutem Alkohol nach, füllt bis zur Marke anf, und schüttelt mehrere Male gut durch.

Nach 6-12 Stunden ist die über dem Tabak stehende Lö. sung klar.

Man pipettirt 100 C.C. heraus und bringt dieselben in einen Kolben mit doppelt durchbohrten Kork. Durch eine Bohrung desselben ist ein Trichterrohr mit feiner Spitze bis auf den Boden des Kolbens eingeführt, die andere enthält nur ein unter dem Kork abschneidendes Rohr, welches mit dem Kühler verbunden wird. 
Man destillirt die Hauptmenge des Alkohols ab, giesst nach dem Erkalten durch das Trichterrohr 30 C.C. einer mässig verdünnten Kalilauge von 1,159 (die Kalilauge der Pharmacopöe wird zur Herstellung dieser Lauge mit dem gleichen Volum Wasser verdünnt) zum Rückstand und destillirt auf dem Sandbad bei mässiger Flamme, bis die übergehenden Tropfen auf Lackmuspapier keine Reaction zeigen.

Nach der Titration mil Zehntel - Normal-Schwefelsäure hat man nur nöthig die gefundenen Cubikcentimeter durch 5 zu dividiren, um die Procente Nicotin des untersuchten Tabaks za erhalten. ${ }^{1}$

\section{Einige neue Reactionen der Milch.}

Von Dr. C. Arnold in Hannover.

Um gekochte von frischer Milch zu unterscheiden, sind wir auf den Geruch und Geschmack derselben angewiesen, was jedoch nur Geübten gelingen dürfte. Die Quevenne'sche Angabe, dass gekochte Milch später und weniger vollständig coagulirt wie ungekochte, ist häufig nicht zutreffend.

Versetzt man frische Milch .mit etwas Guajaktinctur, so tritt sofort oder nach wenig Sekunden eine mehr oder minder intensive Blaufärbung auf, die längere Zeit bestehen bleibt. Mehr wie 20 verschiedene untersuchte Milchsorten gaben die Reaction ohne Ausnahme. Beim vorsichtigen Erwärmen der Milch auf $40-60^{\circ} \mathrm{C}$. tritt die Reaction sofort ein, ebenfalls bei $70-78^{\circ} \mathrm{C}$, aber schwächer, Milch über $80^{\circ}$ erwärmt, bleibt nach Zusatz der Guajaklösung ungefärbt, sowohl in der Wärme wie nach dem Erkalten, ebenso jede einmal aufgekochte Milch. Condensirte Milch zeigt folglich diese Erscheinung gleichfalls nicht. Die Reaction mit Guajaktinctur ist so empfindlich, dass ein Tropfen Milch in einem Uhrglase mit einer Spur obiger Tinctur versetzt, oder ein Tropfen Milch auf Filtrirpapier gebracht und mit einem mit Guajaktinctur benetzten Glasstabe bestrichen, noch blaue Färbung annahmen. Saure Milch

1) Durch Eindampfen des Filtrats und Aufnehmen in $98 \%$ tigen Alkohol überzeugt man sich von der Abwosenheit des Ammoniumsulfats. 